\title{
Mycobacterium ulcerans Disease (Buruli Ulcer): Potential Reservoirs and Vectors
}

\author{
Katharina Röltgen • Gerd Pluschke
}

Published online: 27 January 2015

(C) Springer International Publishing AG 2015

\begin{abstract}
Mycobacterium ulcerans is an emerging pathogen causing the skin infection Buruli ulcer (BU), one of the most neglected tropical diseases. BU is characterized by the formation of chronic, necrotizing skin lesions. This pathology is mainly attributed to the cytotoxic and immunosuppressive activities of the unique polyketide-derived macrolide toxin mycolactone secreted by the pathogen. The disease has been reported from more than 30 countries worldwide, with an extremely focal geographic distribution within endemic countries and highest incidences in remote communities of West/ Central Africa and also certain coastal areas of Australia. While M. ulcerans has long been considered an environmental bacterium, recent findings from southeastern Australia, identifying possums as probable reservoirs of infection, indicate its zoonotic potential. The exact route of $M$. ulcerans transmission is unclear, although it is commonly assumed that infection takes place either through physical contact with environmental reservoirs via skin abrasions or through insect bites, while direct human-to-human transmission seems to be rare.
\end{abstract}

Keywords Mycobacterium ulcerans · Buruli ulcer . Neglected tropical disease $\cdot$ Animal reservoir $\cdot$ Possums

This article is part of the Topical Collection on Bacteriology

K. Röltgen · G. Pluschke $(\bowtie)$

Molecular Immunology Unit, Swiss Tropical and Public Health Institute, Basel, Switzerland

e-mail: Gerd.Pluschke@unibas.ch

K. Röltgen

e-mail: Katharina.Roeltgen@unibas.ch

K. Röltgen · G. Pluschke

University of Basel, Basel, Switzerland

\section{Introduction}

The necrotizing skin disease Buruli ulcer (BU) is characterized by a strikingly focal geographic distribution of cases in the endemic countries [1], yet mechanisms of transmission remain elusive. BU foci in Africa and Australia (Fig. 1a, b, respectively) are typically associated with stagnant and slowflowing water bodies [2], prompting the assumption that infection with the etiological agent Mycobacterium ulcerans occurs through contact with a reservoir in wetland ecosystems. However, definite evidence for the presence of viable M. ulcerans in potential environmental reservoirs is lacking. Until today, environmental studies are mainly based on the detection of the two main features differentiating the genome of M. ulcerans from related species, namely the insertion sequence (IS) elements 2404 and 2606 as well as sequences in the virulence plasmid (pMUM) [3, 4.•] encoding enzymes required for the elaboration of the cytotoxic and immunosuppressive toxin mycolactone. In recent years, mycolactoneproducing mycobacteria (MPM) have been isolated from various hosts [5-8]. Comparative genome analysis suggests that all MPM have diverged from a common Mycobacterium marinum-like ancestor [9] and variants should be classified as ecovars of the species M. ulcerans. M. marinum is a ubiquitous waterborne organism that infects fish and amphibians. Infection in humans occurs only occasionally by inoculation of the bacteria through pre-existing wounds, leading to the development of granulomatous, self-limiting skin lesions. The ability to produce mycolactone by acquisition of the plasmid pMUM can thus be considered as a first step in the emergence of an infectious agent with increased virulence. In the course of its evolution, M. ulcerans diverged into at least two principal lineages $[10,11 \bullet]$. Strains of the so called ancestral lineage typically cause disease in ectotherms, but also sporadically infect humans [12-14]. In contrast, strains of the classical lineage are typically isolated from human BU patients, but 
Fig. 1 Photographs of BU foci in Africa and south-eastern Australia. Landscapes typical for BU endemic regions in Cameroon (a) and Victoria, Australia (b). BU lesions on the arm of a patient (c) and on the head of an infected possum (d). Common ringtail possum (e) and ringtail possum feces (f)
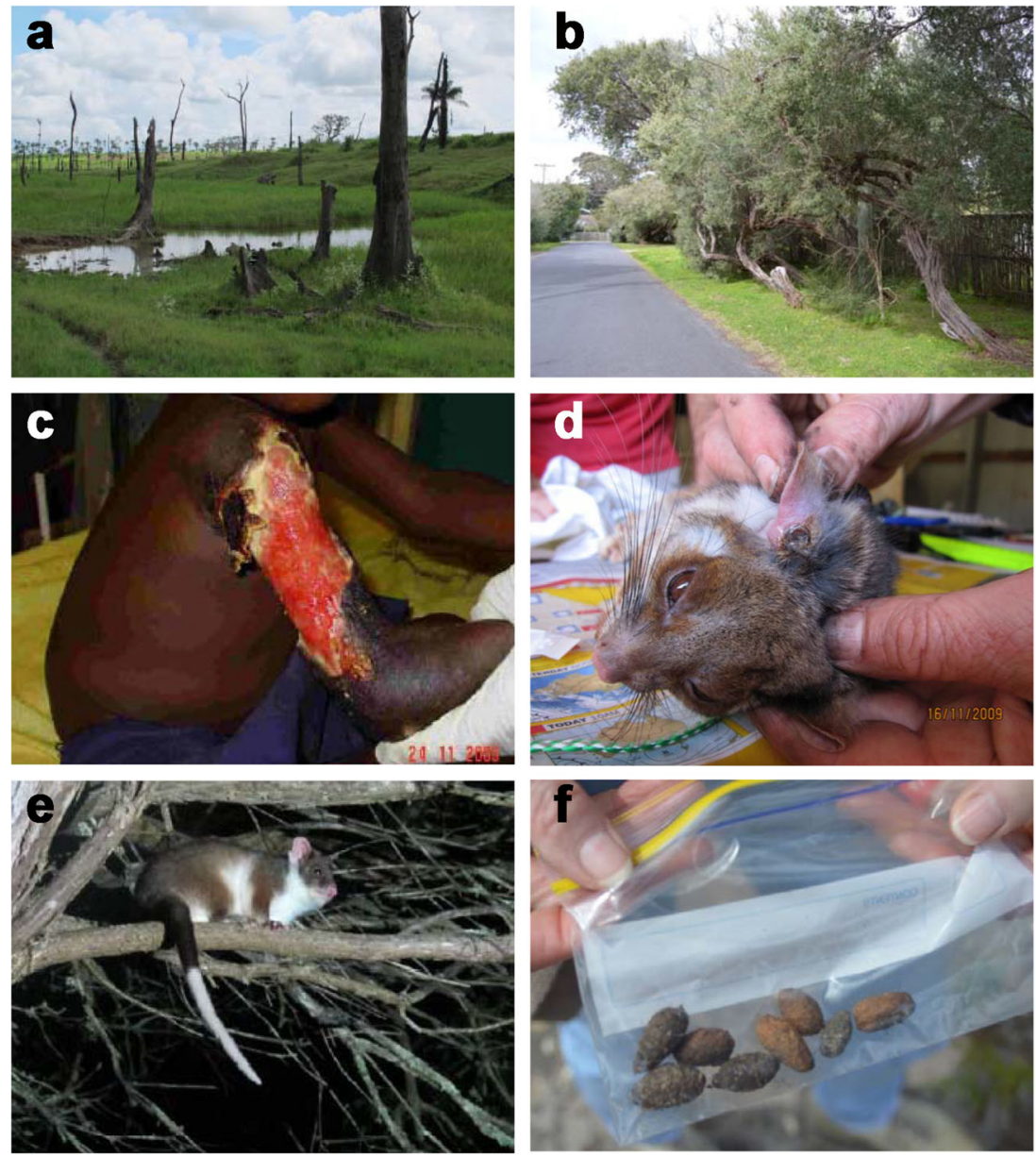

can also infect other endotherms $[10,11 \bullet, 15 \bullet]$. In southeastern Australia infections by $M$. ulcerans strains belonging to the classical lineage were reported in a wide range of terrestrial mammals [16-20], in particular in possums, small arboreal marsupials $[15 \bullet, 21]$. In areas known to be endemic for human BU, significant numbers of possums were shown to have clinical $M$. ulcerans infections, implying their potential as reservoirs for the pathogen $[4 \bullet \bullet, 15 \bullet]$. Until today, a similar animal reservoir has not been identified in African BU endemic areas [22], but it has been suggested that BU patients with large chronic ulcerative lesions may play an active role in the dissemination of M. ulcerans in the environment [23, 24]. However, it remains to be established in which way the pathogen is transmitted from potential environmental sources to humans.

\section{Host Range of $M$. ulcerans}

M. ulcerans was first isolated in 1948 by MacCallum and his colleagues from the skin lesions of BU patients living in an area around Bairnsdale, Australia [25]. In the following decades, M. ulcerans infections have exclusively been associated with BU in humans and disease foci were reported in particular in a number of West and Central African countries [26-31] with highest incidence rates in Benin [32], Ivory Coast [33], Ghana [34], and Cameroon [35].

Early skin manifestations of BU disease appear as relatively unspecific painless nodules, papules, or plaques and are often neglected by the patients. Advanced stages of the disease are characterized by necrosis of subcutaneous adipose tissue and breakdown of the skin, resulting in the formation of slowly progressing, ulcerative skin lesions (Fig. 1c) [1]. The skin is a favorable site of infection, since $M$. ulcerans only grows at temperatures below $37^{\circ} \mathrm{C}$ [36]. While it had long been supposed that the characteristic necrotic pathology is caused by the activity of a toxic substance [37], it was only in 1999 that the polyketide-derived macrolide toxin mycolactone was identified as the major virulence factor of M. ulcerans [38]. The production of mycolactone is peculiar in many respects. For one, mycolactones are the first discovered macrolides in a mycobacterial species and also the first identified polyketide virulence determinants of a bacterial human pathogen [39]. Hence, it has been assumed that the property of producing mycolactone was unique to M. ulcerans, coining the evolution of a new mycobacterial species. It was 
thus all the more surprising when in recent years other MPM have been isolated from different hosts and given various species designations [5-8]. In 2004, MPM have been isolated in a mycobacteriosis outbreak in a laboratory frog colony of Xenopus tropicalis [5]. At that time, the species name Mycobacterium liflandii was proposed for these bacteria, although the taxonomic position had not been fully elucidated [40]. Other MPM have been detected in diseased striped bass from Chesapeake Bay (USA) and designated M. pseudoshottsii [6]. Furthermore, an unusual clade of mycolactone-producing M. marinum has been reported from fish in the Red and Mediterranean Seas [7]. Comparative genomic studies of $M$. ulcerans isolates from human lesions, MPM from other sources and M. marinum isolates suggested that all MPM have evolved from a common M. marinum-like progenitor and represent a single species, which should be designated $M$. ulcerans $[9,11 \bullet, 41]$. Genomic analyses by Doig and colleagues [11•] further indicated a divergence of M. ulcerans into sub-lineages, occupying different ecological niches. Ancestral M. ulcerans strains were divided into lineage 1 including human isolates from South America as well as fish and frog isolates and lineage 2 consisting of clinical M. ulcerans isolates from Japan. Classical M. ulcerans strains - defined as lineage 3-were represented by human isolates from Africa as well as human and animal isolates from Australia. In areas of south-eastern Australia known to be endemic for human BU, M. ulcerans infections have not only been described in different wild possum species [42], but also in other native wildlife and domestic mammals, including koalas [43], horses [17], dogs [18], alpacas [20], and a cat [16]. However, possums appear to be particularly susceptible to M. ulcerans. Common ringtail possums were shown to develop single or multiple ulcerative cutaneous lesions and can become systemically and potentially fatally infected with M. ulcerans [42].

\section{Environmental and Animal Reservoirs of M. ulcerans}

\section{Environmental Reservoirs}

While outbreaks of BU have consistently been connected with stagnant bodies of water or river basins, definite environmental reservoirs could not be established so far. Due to the extremely slow growth rate of M. ulcerans, cultivation from potential environmental sources contaminated with fastergrowing mycobacterial species resistant to decontamination procedures seems to be virtually impossible. Until today, only one report described the isolation of an M. ulcerans strain from an environmental source, an aquatic insect collected in Benin [44], after several passages in mouse footpads. Indirect evidence for the presence of $M$. ulcerans in the environment has been provided by the detection of M. ulcerans-specific
DNA sequences in many biotic components of aquatic ecosystems, such as plants, snails, fish, or insects. However, the existence of M. ulcerans DNA in the environment is not necessarily indicative of a potential reservoir, but may rather point towards the ubiquitous presence of M. ulcerans in these ecosystems [2] and thus results are difficult to interpret. An indication for an aquatic niche environment was given in a recent longitudinal study reporting the persistence of $M$. ulcerans DNA in a village water site located in the BU endemic Mapé basin of Cameroon. Underwater detritus at a localized position of the water hole was repeatedly tested positive for M. ulcerans-specific DNA sequences over a period of more than 2 years after all local BU cases had been treated [45], suggesting that $M$. ulcerans has adapted to survive in a restricted niche environment. Several studies have indicated that M. ulcerans is unlikely to be free-living and may persist in the environment as a commensal, associated with another protective organism such as amoeboid protozoa or inhabitants of biofilms in stagnant water bodies [46-48]. Characteristic genome signatures of $M$. ulcerans including (1) the acquisition of foreign DNA such as pMUM, IS2404, and IS2606, (2) the proliferation of IS elements, (3) extensive gene loss through pseudogene formation, and (4) genome downsizing, provide further evidence that $M$. ulcerans is adapting to a new, probably relatively protected and stable ecological niche $[11 \bullet, 49$, 50]. Deletion or inactivation of genes required for pigment biosynthesis and anaerobiosis led to the assumption that M. ulcerans is adapting to a dark and aerobic environment. Evolving M. ulcerans sub-lineages seem to adapt to related but distinct niche environments and should be considered ecovars of the same species. Additional loss of gene function among the highly clonal sub-lineage 3 strains isolated from human BU cases in Africa and Australia indicates that this lineage has passed through another evolutionary bottleneck and has further adapted to live in a highly restricted niche environment [11•].

\section{Animal Reservoirs}

In south-eastern Australia, a series of focal BU outbreaks in the last two decades has puzzled researchers, struggling to identify reservoirs and vectors of the pathogen. A major breakthrough was achieved in 2010, when systematic testing of environmental specimens from BU endemic areas revealed the highest concentrations of M. ulcerans DNA in the feces of possums (Fig. 1f), small tree-dwelling marsupials (Fig. 1e). Examination of these animals showed that a large proportion of them had developed M. ulcerans skin lesions (Fig. 1d), indicating their potential as infectious reservoir host. In support of these findings, genome comparison of M. ulcerans isolates from a ringtail possum and a human patient from the same BU endemic region revealed an extremely close genetic relationship with only two identified single nucleotide 
polymorphisms [15•]. Moreover, a strong positive correlation was observed between the prevalence of $M$. ulcerans DNA in possum feces and BU endemicity of an area $[4 \bullet \bullet]$.

In African BU endemic regions, surveys of small animals have so far not led to the identification of any species with BU lesions or viable bacteria in their feces [22, 51]. However, there is some evidence that $\mathrm{BU}$ patients with ulcerative lesions may play an active role in the dissemination and distribution of the pathogen in the environment. For one, it has recently been reported that genetic variants of M. ulcerans occur in focal transmission clusters within individual BU endemic areas and are not readily spread between geographically separated endemic areas $[23,52]$. Also, in a BU focus of Benin, it has recently been observed that a positive correlation exists between the BU endemicity of a region and the presence of M. ulcerans DNA in the environment [24].

\section{Potential Vectors}

Memory of patients regarding the beginning of a BU lesion or the potential causative event of infection is compromised by the highly variable and extremely long incubation period of several months [53]. While it is generally assumed that M. ulcerans is acquired through environmental contacts, different routes of infection with the pathogen have been controversially discussed. These include inoculation of the bacteria via skin trauma or pre-existing wounds from an environmental reservoir, or via bites from insects, such as water bugs or mosquitoes. Considerable efforts have been undertaken in the past decades to identify vectors of M. ulcerans and to unravel the exact mode of transmission by various approaches.

One of these strategies was to examine the distribution pattern of BU lesions on the body, since in patients with single lesions, the site of lesion is assumed to be the site of inoculation. Many different reports from Africa and a study in Australia have consistently shown that the majority of lesions is located on the lower limbs [21, 32, 34, 54-56] and a focus on joints has been described in adults $[21,55,56]$. Furthermore, the anatomical site of the lesions was shown to vary with both age and gender. While children tend to have a more dispersed distribution of lesions over the body, lesions seem to become increasingly confined to the limbs in adults $[32,45,54,57]$, presumably because children cover their trunks less often compared to adults. The gender difference varies between studies and is thought to be influenced by behavioral factors and environmental contact patterns. Thus, in a highly BU endemic community of Rwandan refugees living in central Uganda in the 1960s, where males carried out agricultural activities using hoes or other tools, whereas females generally worked with only their hands, lesions in males were restricted to the lower legs, while in females the arms were also often affected [57]. Along these lines, lesions on the trunk were found to be less common in females, which are more likely to cover their upper body with clothing [56]. While results of all of these studies showed that lesions occur mostly at locations where the skin is not commonly protected with clothing, a definite mode of transmission could not be established, since the distribution of $\mathrm{BU}$ lesions did neither match reported mosquito biting patterns, nor seemed to correlate with the published distribution of small skin injuries in children $[56,58]$.

Another strategy to give some indication of possible vectors and ways of transmission was to analyze a potential association between age and exposure to M. ulcerans. A recent survey for BU in Cameroon has revealed a bimodal age distribution with increased incidence in young persons and the elderly, while very young children were underrepresented among cases when taking the overall population age distribution into account [56]. In that vein, findings from our seroepidemiological studies, where we determined antibody titers against the $18-\mathrm{kDa}$-small heat shock protein of M. ulcerans in healthy individuals living in BU endemic areas of Cameroon and Ghana, suggested that children below the age of 5 years are considerably less exposed to the pathogen than older children. This indicates that exposure to M. ulcerans and thus infection with the pathogen might occur outside of the relatively small movement range of very young children and increases in older children with more direct environmental contacts further away from their homes [59]. Hence, in African, BU endemic settings infection through vectors commonly present in the limited movement radius of young children appears unlikely.

Another intriguing aspect of these seroepidemiological investigations in $\mathrm{BU}$ endemic regions is that exposure to M. ulcerans in many cases seems to lead to nonsymptomatic infections [59,60]. Factors accounting for this diverse outcome of infection may be manifold, as observed also for other mycobacterial diseases such as tuberculosis and leprosy. Strong evidence that the inoculation dose of M. ulcerans may be critical for the outcome of an infection was recently provided by experimental infection of pigs. While high doses of M. ulcerans led to the development of BU lesions, infiltrating immune cells appeared to cope well with lower doses of bacteria, which were completely eliminated after a couple of weeks [61]. M. ulcerans clusters of a critical size may establish a chronic infection focus by the development of a protective cloud of mycolactone around them, killing infiltrating cells before they can clear the bacteria. In this context, it remains to be investigated whether the location of $M$. ulcerans clusters, which are typically found deep in the subcutaneous fat tissue of BU lesions, may be related to the mode of transmission or merely reflect the preferred habitat of the bacteria. In a study by Williamson et al., 
alternative hypotheses regarding $M$. ulcerans transmission were tested in a guinea pig infection model. While intradermal injection of $M$. ulcerans consistently led to the development of an ulcer, topical application of the bacteria to an open abrasion failed to establish an infection. M. ulcerans could no longer be isolated from abrasions $48 \mathrm{~h}$ post inoculation, whereas cultures could be obtained from injection sites over extended periods of time. These data indicate that BU lesions are unlikely to be caused by entry of the bacteria via abrasions and rather speak for a requirement of biting invertebrates, puncture wounds, or lacerations [62].

Following the report of the successful isolation of M. ulcerans from an aquatic insect collected in Benin [44], several studies have investigated the potential of invertebrate species to serve as vectors of M. ulcerans in Africa, but this concept has remained controversial. Results from laboratory experiments have shown that $M$. ulcerans can survive in the salivary glands of different invertebrate species and moreover, experimental transmission from an invertebrate vector to a mouse has been demonstrated [63]. However, direct infection through biting water bugs may only occur accidently, mainly because the invertebrate species that have been associated with the transmission of M. ulcerans are not hematophagous [2]. Hence, it was suggested that aquatic insects may rather play a role in maintaining $M$. ulcerans within food webs in the environment [64, 65].

In south-eastern Australia, different studies have indicated an involvement of mosquitoes in the transmission of the disease. For one, a case-control study carried out during a BU outbreak in 2007 reported increased odds of having BU for those who were bitten by mosquitoes on the lower extremities [66]. Furthermore, M. ulcerans-specific DNA sequences were detected in the same year in mosquitoes captured from the BU endemic area [67] and more recently an association between the incidence of human BU in several communities and the proportion of $M$. ulcerans-positive mosquitoes captured in the same locations was found [68]. However, it is still not known, whether mosquitoes may act as biological vectors of M. ulcerans or only passively carry the bacteria on their surface. Laboratory experiments showing that M. ulcerans is readily ingested by mosquito larvae, but not maintained throughout the mosquito developmental cycle into pupal or adult stages [69], speak against their role as biological vectors.

Taken together, no single vector or transmission pathway could be identified so far, which encourages the hypothesis that multiple modes of transmission may exist $[54,56]$.

\section{Proposed Transmission Model(s) and Potential Zoonotic Risk}

A major obstacle in quest of the mode of M. ulcerans transmission has long been the attempt to find a single, plain cause of infection. In recent years, a process of rethinking has begun, acknowledging that infection with $M$. ulcerans may occur through diverse modes, involving various environmental and/or animal reservoirs and possibly even different vectors. While M. ulcerans ecovars of the ancestral lineage are adapting to an ecological niche environment, from which they appear to only accidently infect humans, strains of the highly clonal classical lineage are responsible for human BU disease foci in Australia and Africa, representing the majority of the global BU burden. Comparative studies of M. ulcerans genomes have furthermore revealed that a strikingly close relationship exists between genotype and geographical origin of isolates, implying that M. ulcerans transmission is highly focal and therefore reservoir(s) and/or vector(s) seem to be geographically fixed $[11 \bullet, 23,52]$. This is consistent with the finding that BU endemic and non-endemic villages are often separated by only a few kilometers [60].

In south-eastern Australia, the significant BU burden of possums in geographically restricted regions suggests a zoonotic infection reservoir. In the BU endemic community Point Lonsdale clinical examination of possums between 2008 and 2009 revealed that $38 \%$ of ringtail possums and $24 \%$ of brushtail possums had laboratory-confirmed BU lesions and/ or M. ulcerans PCR-positive feces. However, the way in which $M$. ulcerans might be transmitted from possums to humans is not clear. Striking similarities exist between the epidemiology of BU and zoonotic plague in Madagascar caused by Yersinia pestis. This pathogen is likewise characterized by limited genetic diversity and genotypes equally appear to be maintained in geographically separated subpopulations. Similar to the transmission model established for $Y$. pestis, where the geographic-genetic pattern is explained by the natural cycle between its primary host, the black rat, and its flea vectors [70], a concept involving possums as possible reservoir and mosquitoes as potential vectors has been proposed for M. ulcerans in south-east Australian BU endemic settings. In this model, possums may take up M. ulcerans from environmental sources or may be infected by an insect vector and pass the bacteria back into the environment. Transmission to humans is thought to occur via biting mosquitoes, which had either direct contact to the lesion of a diseased possum or rested on vegetation contaminated by a possum lesion. Although the viability of the pathogen in possum feces remains to be demonstrated, it may alternatively be envisaged that mosquitoes take up the bacteria through contact with environments contaminated by possum feces containing $M$. ulcerans. Along these lines, an intriguing parallel was drawn between the epidemiology of BU and leptospirosis by Fyfe et al. Leptospirosis, a zoonosis of worldwide distribution, is transmitted through contact with environments contaminated by the urine of infected animals [15•].

While until today, no animal reservoir for M. ulcerans has been identified in Africa, it has been hypothesized that 
humans with active BU lesions play an important role in the spread of the bacteria into the environment. This is supported by the decline of BU incidence recorded in some areas with improved BU surveillance and early treatment. Contrary to the BU situation in Australia, where patients usually seek treatment at early stages of the disease, limited access to health facilities, economic limitations, traditional beliefs, and stigmatization often lead to a drastic treatment delay in Africa, with patients suffering for many months or years from large active ulcerative lesions, shedding the bacteria into the environment. Since recent studies indicated that $M$. ulcerans may persist for many months in under water decaying organic matter [45], infection may occur at contaminated water contact sites. In other studies, a conceptual model has been proposed, where M. ulcerans, present in the aquatic environment such as in detritus, mud, or plant biofilms, is concentrated by waterfiltering organisms and passed on to predatory aquatic vertebrates and invertebrates feeding on this prey $[2,71]$. Transmission may take place through puncture wounds or lacerations after contact with concentrated M. ulcerans sources or occasionally via invertebrate vectors.

\section{Risk Factors and Control}

Until now, the diversity of possible M. ulcerans reservoirs and potential mechanisms of infection has made it impossible to unambiguously identify preventable risks of infection. However, better understanding of transmission may in future allow improving control of the disease by modifying behavioral patterns and environmental features. Since no BU vaccine is presently available, early case detection and rapid initiation of the recommended 8-week course of daily rifampicin-streptomycin antibiotic treatment are key elements to prevent long-term disability as well as to interrupt the shedding of bacteria from active lesions into the environment. Results from several risk factor studies for BU conducted in Australia and different African countries were systematically compared and evaluated in a review article published in 2010. The most common risk factors identified on both continents were poor wound care, failure to wear protective clothing, and living or working near water bodies [72]. An additional risk factor determined in a case-control study conducted in southeastern Australia was exposure to mosquitoes. The authors reported that more cases than control individuals recalled that they were bitten by mosquitoes on the lower extremities and that the use of insect repellent conferred reduced risk of getting BU [66]. Even where risk factors have been identified, practical implementation of recommendations for behavioral changes may be challenging. Accordingly, the most desired solution to control BU in focal, highly BU endemic areas would be the targeted vaccination of populations at risk. However, despite efforts to develop novel vaccine strategies
[73], no vaccine candidate is currently in advanced development.

Vaccine development is hampered by incomplete knowledge of the nature of immune defense mechanisms needed to control the infection. In this regard, it is debated, whether M. ulcerans should be classified as extracellular or intracellular pathogen, which has major implications for vaccine design. While protective adaptive immunity against extracellular pathogens relies primarily on the generation of functional antibodies, immune protection against intracellular pathogens is typically achieved by cell-mediated immunity. While M. ulcerans has long been considered as being primarily extracellular, it has been shown recently that it also owns essential hallmarks of an intracellular organism leading to infectionassociated development of cell-mediated immune responses and delayed-type hypersensitivity [74]. There is evidence for the existence of a transient intracellular stage of M. ulcerans during the establishment of an infection [74-76]. In more advanced stages, mycolactone is thought to mediate the killing of host cells and infiltrating leukocytes. Subsequent extracellular multiplication of the bacteria, producing a protective cloud of mycolactone, leads to the characteristic histopathology of clumps of extracellular bacteria surrounded by necrosis of the deep dermal and adipose tissue associated with limited inflammatory response [77]. Future research should be targeted at unraveling protective immune defense mechanisms against $M$. ulcerans and the development of improved vaccination strategies, including mycolactone-targeting vaccines, attenuated live vaccines or subunit protein vaccines.

In south-eastern Australia, new M. ulcerans infection foci are frequently emerging in close proximity to established hotspots. In the emergent BU outbreak area located in the Mornington Peninsula, it has been investigated whether systematic testing of ground collected possum feces for the presence of M. ulcerans DNA can be used as a tool to predict the geographic shift of endemic areas. The striking correlation between M. ulcerans-positive possum feces in areas, where human BU has been reported, combined with the relative absence of M. ulcerans DNA in possum feces adjacent to these areas indicates that possums may be useful sentinels to monitor the emergence, and spread of human BU in south-eastern Australia for public health planning [4*0]. This approach has been described for the surveillance of other zoonotic diseases such as West Nile virus or Lyme disease, assessed by diagnostic testing of wild birds, which are known to maintain and transmit many zoonotic pathogens [78]. However, the temporal relationship between the presence of $M$. ulcerans DNA in possums and human BU outbreaks in the same area is yet to be established. A study by Carson et al. has suggested that the relative population density of the two mainly affected possum species, ringtail, and brushtail possums may influence endemicity and emergence of human BU [4••]. A survey of the possum population dynamics in endemic and non-endemic 
areas is needed to support the hypothesis regarding the necessity for a coexistence of both species in the outbreak area. It remains to be established if and how information on the possum populations can be used to support the control of BU.

\section{Conclusion}

In spite of considerable research efforts in the past decades, no conclusive explanation for infection with the emerging pathogen M. ulcerans could be defined, leading to the assumption that multiple modes of transmission may exist. Due to the lack of effective prevention strategies, the present approach to control BU relies on active case search in regions suspected to be endemic and subsequent antibiotic treatment. Both in Africa and in Australia BU is characterized by highly focal transmission clusters. In south-eastern Australia, the occurrence of BU in localized regions has been associated with the infection of possums and consequently M. ulcerans is considered a potential zoonotic pathogen in this region. Future research should be directed at the identification of animal reservoirs for M. ulcerans in African settings and at the verification of the proposed modes of transmission.

Acknowledgements We thank Dr. Janet Fyfe, Dr. Kath Handasyde, and Dr. Carolyn O'Brien for providing photographs of possums with BU lesion.

\section{Compliance with Ethics Guidelines}

Conflict of Interest Dr. Pluschke and Dr. Röltgen both declare they have no conflict of interest.

Human and Animal Rights and Informed Consent This article contains no studies with human or animal subjects performed by any of the authors.

\section{References}

Papers of particular interest, published recently, have been highlighted as:

- Of importance

•- Of major importance

1. Junghanss T, Johnson RC, Pluschke G. Mycobacterium ulcerans disease. In: Farrar J, Hotez PJ, Junghanss T, Kang G, Lalloo D, White N, editors. Manson's tropical diseases. 23rd ed. Edinburgh: Saunders; 2014. p. 519-31.

2. Merritt RW, Walker ED, Small PLC, Wallace JR, Johnson PDR, Benbow ME, et al. Ecology and transmission of Buruli ulcer disease: a systematic review. PLoS Negl Trop Dis. 2010;4:e911. doi: 10.1371/journal.pntd.0000911.

3. Fyfe JAM, Lavender CJ, Johnson PDR, Globan M, Sievers A, Azuolas J, et al. Development and application of two multiplex real-time PCR assays for the detection of Mycobacterium ulcerans in clinical and environmental samples. Appl Environ Microbiol. 2007;73:4733-40. doi:10.1128/AEM. 02971-06.

4.• Carson C, Lavender CJ, Handasyde KA, O’Brien CR, Hewitt N, Johnson PDR, et al. Potential wildlife sentinels for monitoring the endemic spread of human buruli ulcer in South-East australia. PLoS Negl Trop Dis. 2014;8:e2668. doi:10.1371/journal.pntd.0002668. This study conducted in south-eastern Australia revealed an association of human BU cases with areas where M. ulcerans positive possum faeces are found, providing further evidence for a potential role of possums as an environmental reservoir of infection. The authors suggested that possums may be used as sentinels to monitor human BU in south-eastern Australia.

5. Trott KA, Stacy BA, Lifland BD, Diggs HE, Harland RM, Khokha MK, et al. Characterization of a Mycobacterium ulcerans-like infection in a colony of African tropical clawed frogs (Xenopus tropicalis). Comp Med. 2004;54:309-17.

6. Rhodes MW, Kator H, McNabb A, Deshayes C, Reyrat J-M, Brown-Elliott BA, et al. Mycobacterium pseudoshottsii sp. nov., a slowly growing chromogenic species isolated from Chesapeake Bay striped bass (Morone saxatilis). Int J Syst Evol Microbiol. 2005;55:1139-47. doi:10.1099/ijs. 0.63343-0.

7. Ranger BS, Mahrous EA, Mosi L, Adusumilli S, Lee RE, Colorni A, et al. Globally distributed mycobacterial fish pathogens produce a novel plasmid-encoded toxic macrolide, mycolactone F. Infect Immun. 2006;74:6037-45. doi:10.1128/IAI. 00970-06.

8. Stragier P, Hermans K, Stinear T, Portaels F. First report of a mycolactone-producing Mycobacterium infection in fish agriculture in Belgium. FEMS Microbiol Lett. 2008;286:93-5. doi:10. 1111/j.1574-6968.2008.01264.x.

9. Yip MJ, Porter JL, Fyfe JAM, Lavender CJ, Portaels F, Rhodes M, et al. Evolution of Mycobacterium ulcerans and other mycolactoneproducing mycobacteria from a common Mycobacterium marinum progenitor. J Bacteriol. 2007;189:2021-9. doi:10.1128/JB. 01442-06.

10. Käser M, Rondini S, Naegeli M, Stinear T, Portaels F, Certa U, et al. Evolution of two distinct phylogenetic lineages of the emerging human pathogen Mycobacterium ulcerans. BMC Evol Biol. 2007;7:177. doi:10.1186/1471-2148-7-177.

11. Doig KD, Holt KE, Fyfe JAM, Lavender CJ, Eddyani M, Portaels F, et al. On the origin of Mycobacterium ulcerans, the causative agent of Buruli ulcer. BMC Genomics. 2012;13:258. doi:10.1186/14712164-13-258. This comparative genome analysis revealed that $M$. ulcerans and all mycolactone-producing mycobacteria are specialized variants of a common M. marinum-like ancestor that have adapted to live in restricted environments.

12. Faber WR, Arias-Bouda LM, Zeegelaar JE, Kolk AH, Fonteyne PA, Toonstra J, et al. First reported case of Mycobacterium ulcerans infection in a patient from China. Trans R Soc Trop Med Hyg. 2000;94:277-9.

13. Guerra H, Palomino JC, Falconí E, Bravo F, Donaires N, Van Marck E, et al. Mycobacterium ulcerans disease, Peru. Emerg Infect Dis. 2008;14:373-7. doi:10.3201/eid1403.070904.

14. Nakanaga K, Hoshino Y, Yotsu RR, Makino M, Ishii N. Nineteen cases of Buruli ulcer diagnosed in Japan from 1980 to 2010. J Clin Microbiol. 2011;49:3829-36. doi:10.1128/JCM. 00783-11.

15. Fyfe JAM, Lavender CJ, Handasyde KA, Legione AR, O'Brien CR, Stinear TP, et al. A major role for mammals in the ecology of Mycobacterium ulcerans. PLoS Negl Trop Dis. 2010;4:e791. doi: 10.1371/journal.pntd.0000791. These authors proposed a transmission model for $B U$, in which possums are implicated as reservoirs for M. ulcerans.

16. Elsner L, Wayne J, O'Brien CR, McCowan C, Malik R, Hayman JA, et al. Localised Mycobacterium ulcerans infection in a cat in Australia. J Feline Med Surg. 2008;10:407-12. doi:10.1016/j.jfms. 2008.03.003. 
17. Van Zyl A, Daniel J, Wayne J, McCowan C, Malik R, Jelfs P, et al. Mycobacterium ulcerans infections in two horses in south-eastern Australia. Aust Vet J. 2010;88:101-6. doi:10.1111/j.1751-0813. 2009.00544.x.

18. O'Brien CR, McMillan E, Harris O, O'Brien DP, Lavender CJ, Globan M, et al. Localised Mycobacterium ulcerans infection in four dogs. Aust Vet J. 2011;89:506-10. doi:10.1111/j.1751-0813. 2011.00850.x.

19. Malik R, Smits B, Reppas G, Laprie C, O’Brien C, Fyfe J. Ulcerated and nonulcerated nontuberculous cutaneous mycobacterial granulomas in cats and dogs. Vet Dermatol. 2013;24:146-53. doi:10.1111/j.1365-3164.2012.01104.x. e32-3.

20. O'Brien C, Kuseff G, McMillan E, McCowan C, Lavender C, Globan M, et al. Mycobacterium ulcerans infection in two alpacas. Aust Vet J. 2013;91:296-300. doi:10.1111/avj.12071.

21. Boyd SC, Athan E, Friedman ND, Hughes A, Walton A, Callan P, et al. Epidemiology, clinical features and diagnosis of Mycobacterium ulcerans in an Australian population. Med J Aust. 2012;196:341-4.

22. Vandelannoote K, Durnez L, Amissah D, Gryseels S, Dodoo A, Yeboah S, et al. Application of real-time PCR in Ghana, a Buruli ulcer-endemic country, confirms the presence of Mycobacterium ulcerans in the environment. FEMS Microbiol Lett. 2010;304: 191-4. doi:10.1111/j.1574-6968.2010.01902.x.

23. Röltgen K, Qi W, Ruf M-T, Mensah-Quainoo E, Pidot SJ, Seemann $\mathrm{T}$, et al. Single nucleotide polymorphism typing of Mycobacterium ulcerans reveals focal transmission of buruli ulcer in a highly endemic region of Ghana. PLoS Negl Trop Dis. 2010;4:e751. doi:10. 1371/journal.pntd.0000751.

24. Williamson HR, Benbow ME, Campbell LP, Johnson CR, Sopoh $\mathrm{G}$, Barogui Y, et al. Detection of Mycobacterium ulcerans in the environment predicts prevalence of Buruli ulcer in Benin. PLoS Negl Trop Dis. 2012;6:e1506. doi:10.1371/journal.pntd.0001506.

25. MacCALLUM P, Tolhurst JC. A new mycobacterial infection in man. J Pathol Bacteriol. 1948;60:93-122.

26. Clancey J, Dodge R, Lunn HF. Study of a mycobacterium causing skin ulceration in Uganda. Ann Soc Belg Med Trop. 1962;42:585-90.

27. Smith JH. Epidemiologic observations on cases of Buruli ulcer seen in a hospital in the Lower Congo. Am J Trop Med Hyg. 1970;19: 657-63.

28. Uganda Buruli Group. Epidemiology of Mycobacterium ulcerans infection (Buruli ulcer) at Kinyara, Uganda. Trans R Soc Trop Med Hyg. 1971;65:763-75.

29. Oluwasanmi JO, Solankee TF, Olurin EO, Itayemi SO, Alabi GO, Lucas AO. Mycobacterium ulcerans (Buruli) skin ulceration in Nigeria. Am J Trop Med Hyg. 1976;25:122-8.

30. Meyers WM, Tignokpa N, Priuli GB, Portaels F. Mycobacterium ulcerans infection (Buruli ulcer): first reported patients in Togo. Br J Dermatol. 1996;134:1116-21.

31. Bär W, Rüsch-Gerdes S, Richter E, Marquéz de Bär G, Dittmer C, Papsdorf $\mathrm{H}$, et al. Mycobacterium ulcerans infection in a child from Angola: diagnosis by direct detection and culture. Trop Med Int Health. 1998;3:189-96.

32. Debacker M, Aguiar J, Steunou C, Zinsou C, Meyers WM, Guédénon A, et al. Mycobacterium ulcerans disease (Buruli ulcer) in rural hospital, Southern Benin, 1997-2001. Emerg Infect Dis. 2004;10:1391-8. doi:10.3201/eid1008.030886.

33. Marston BJ, Diallo MO, Horsburgh CR, Diomande I, Saki MZ, Kanga JM, et al. Emergence of Buruli ulcer disease in the Daloa region of Cote d'Ivoire. Am J Trop Med Hyg. 1995;52:219-24.

34. Amofah G, Bonsu F, Tetteh C, Okrah J, Asamoa K, Asiedu K, et al. Buruli ulcer in Ghana: results of a national case search. Emerg Infect Dis. 2002;8:167-70. doi:10.3201/eid0802.010119.
35. Noeske J, Kuaban C, Rondini S, Sorlin P, Ciaffi L, Mbuagbaw J, et al. Buruli ulcer disease in Cameroon rediscovered. Am J Trop Med Hyg. 2004;70:520-6.

36. Eddyani M, Portaels F. Survival of Mycobacterium ulcerans at $37{ }^{\circ} \mathrm{C}$. Clin Microbiol Infect. 2007;13:1033-5. doi:10.1111/j. 1469-0691.2007.01791.x.

37. Connor DH, Lunn HF. Mycobacterium ulcerans infection (with comments on pathogenesis). Int J Lepr. 1965;33(Suppl):698-709.

38. George KM, Chatterjee D, Gunawardana G, Welty D, Hayman J, Lee R, et al. Mycolactone: a polyketide toxin from Mycobacterium ulcerans required for virulence. Science. 1999;283:854-7.

39. Rohr J. A new role for polyketides. Angew Chem Int Ed Engl. 2000;39:2847-9.

40. Mve-Obiang A, Lee RE, Umstot ES, Trott KA, Grammer TC, Parker JM, et al. A newly discovered mycobacterial pathogen isolated from laboratory colonies of Xenopus species with lethal infections produces a novel form of mycolactone, the Mycobacterium ulcerans macrolide toxin. Infect Immun. 2005;73:3307-12. doi:10. 1128/IAI. 73.6.3307-3312.2005.

41. Pidot SJ, Asiedu K, Käser M, Fyfe JAM, Stinear TP. Mycobacterium ulcerans and other mycolactone-producing mycobacteria should be considered a single species. PLoS Negl Trop Dis. 2010;4:e663. doi:10.1371/journal.pntd.0000663.

42. O'Brien CR, Handasyde KA, Hibble J, Lavender CJ, Legione AR, McCowan C, et al. Clinical, microbiological and pathological findings of Mycobacterium ulcerans infection in three Australian Possum species. PLoS Negl Trop Dis. 2014;8:e2666. doi:10. 1371/journal.pntd.0002666.

43. Mitchell PJ, Jerrett IV, Slee KJ. Skin ulcers caused by Mycobacterium ulcerans in koalas near Bairnsdale, Australia. Pathology. 1984;16:256-60.

44. Portaels F, Meyers WM, Ablordey A, Castro AG, Chemlal K, de Rijk P, et al. First cultivation and characterization of Mycobacterium ulcerans from the environment. PLoS Negl Trop Dis. 2008;2:e178. doi:10.1371/journal.pntd.0000178.

45. Bratschi MW, Ruf M-T, Andreoli A, Minyem JC, Kerber S, Wantong FG, et al. Mycobacterium ulcerans persistence at a village water source of Buruli ulcer patients. PLoS Negl Trop Dis. 2014;8: e2756. doi:10.1371/journal.pntd.0002756.

46. Eddyani M, De Jonckheere JF, Durnez L, Suykerbuyk P, Leirs H, Portaels F. Occurrence of free-living amoebae in communities of low and high endemicity for Buruli ulcer in southern Benin. Appl Environ Microbiol. 2008;74:6547-53. doi:10. 1128/AEM. 01066-08.

47. Gryseels S, Amissah D, Durnez L, Vandelannoote K, Leirs H, De Jonckheere J, et al. Amoebae as potential environmental hosts for Mycobacterium ulcerans and other mycobacteria, but doubtful actors in Buruli ulcer epidemiology. PLoS Negl Trop Dis. 2012;6: e1764. doi:10.1371/journal.pntd.0001764.

48. Amissah NA, Gryseels S, Tobias NJ, Ravadgar B, Suzuki M, Vandelannoote $\mathrm{K}$, et al. Investigating the role of free-living amoebae as a reservoir for mycobacterium ulcerans. PLoS Negl Trop Dis 2014;8. doi:10.1371/journal.pntd.0003148.

49. Stinear TP, Seemann T, Pidot S, Frigui W, Reysset G, Garnier T, et al. Reductive evolution and niche adaptation inferred from the genome of Mycobacterium ulcerans, the causative agent of Buruli ulcer. Genome Res. 2007;17:192-200. doi:10.1101/gr.5942807.

50. Qi W, Käser M, Röltgen K, Yeboah-Manu D, Pluschke G. Genomic diversity and evolution of Mycobacterium ulcerans revealed by next-generation sequencing. PLoS Pathog. 2009;5:e1000580. doi: 10.1371/journal.ppat.1000580.

51. Durnez L, Suykerbuyk P, Nicolas V, Barrière P, Verheyen E, Johnson CR, et al. Terrestrial small mammals as reservoirs of Mycobacterium ulcerans in benin. Appl Environ Microbiol. 2010;76:4574-7. doi:10.1128/AEM. 00199-10. 
52. Vandelannoote K, Jordaens K, Bomans P, Leirs H, Durnez L, Affolabi D, et al. Insertion sequence element single nucleotide polymorphism typing provides insights into the population structure and evolution of Mycobacterium ulcerans across Africa. Appl Environ Microbiol. 2014;80:1197-209. doi:10.1128/AEM. 02774-13.

53. Trubiano JA, Lavender CJ, Fyfe JAM, Bittmann S, Johnson PDR. The incubation period of Buruli ulcer (Mycobacterium ulcerans infection). PLoS Negl Trop Dis. 2013;7:e2463. doi:10.1371/ journal.pntd.0002463.

54. Hospers IC, Wiersma IC, Dijkstra PU, Stienstra Y, Etuaful S, Ampadu EO, et al. Distribution of Buruli ulcer lesions over body surface area in a large case series in Ghana: uncovering clues for mode of transmission. Trans R Soc Trop Med Hyg. 2005;99:196201. doi:10.1016/j.trstmh.2004.05.004.

55. Adu E, Ampadu E, Acheampong D. Surgical management of buruli ulcer disease: a four-year experience from four endemic districts in Ghana. Ghana Med J. 2011;45:4-9.

56. Bratschi MW, Bolz M, Minyem JC, Grize L, Wantong FG, Kerber $\mathrm{S}$, et al. Geographic distribution, age pattern and sites of lesions in a cohort of Buruli ulcer patients from the Mapé Basin of Cameroon. PLoS Negl Trop Dis. 2013;7:e2252. doi:10.1371/journal.pntd. 0002252 .

57. Uganda Buruli Group. Epidemiology of Mycobacterium ulcerans infection (buruli ulcer) at Kinyara, Uganda. Trans R Soc Trop Med Hyg. 1971;65:763-75. doi:10.1016/0035-9203(71)90090-3.

58. De Jong R, Knols BG. Selection of biting sites by mosquitoes. CIBA Found Symp. 1996;200:89-100. discussion 101-8.

59. Röltgen K, Bratschi MW, Ross A, Aboagye SY, Ampah KA, Bolz $\mathrm{M}$, et al. Late onset of the serological response against the $18 \mathrm{kDa}$ small heat shock protein of Mycobacterium ulcerans in children. PLoS Negl Trop Dis. 2014;8:e2904. doi:10.1371/journal.pntd. 0002904

60. Yeboah-Manu D, Röltgen K, Opare W, Asan-Ampah K, QueninFosu K, Asante-Poku A, et al. Sero-epidemiology as a tool to screen populations for exposure to Mycobacterium ulcerans. PLoS Negl Trop Dis. 2012;6:e1460. doi:10.1371/journal.pntd.0001460.

61. Bolz M, Ruggli N, Ruf M-T, Ricklin ME, Zimmer G, Pluschke G. Experimental infection of the pig with Mycobacterium ulcerans: a novel model for studying the pathogenesis of Buruli ulcer disease. PLoS Negl Trop Dis. 2014;8:e2968. doi:10.1371/journal.pntd. 0002968.

62. Williamson HR, Mosi L, Donnell R, Aqqad M, Merritt RW, Small PLC. Mycobacterium ulcerans fails to infect through skin abrasions in a guinea pig infection model: implications for transmission. PLoS Negl Trop Dis. 2014;8:e2770. doi:10.1371/journal.pntd. 0002770.

63. Marsollier L, Robert R, Aubry J, Saint André J-P, Kouakou H, Legras $\mathrm{P}$, et al. Aquatic insects as a vector for Mycobacterium ulcerans. Appl Environ Microbiol. 2002;68:4623-8.

64. Benbow ME, Williamson H, Kimbirauskas R, McIntosh MD, Kolar R, Quaye C, et al. Aquatic invertebrates as unlikely vectors of Buruli ulcer disease. Emerg Infect Dis. 2008;14:1247-54. doi: 10.3201/eid1408.071503.
65. Mosi L, Williamson H, Wallace JR, Merritt RW, Small PLC. Persistent association of Mycobacterium ulcerans with West African predaceous insects of the family belostomatidae. Appl Environ Microbiol. 2008;74:7036-42. doi:10.1128/AEM. 01234-08.

66. Quek TYJ, Athan E, Henry MJ, Pasco JA, Redden-Hoare J, Hughes A, et al. Risk factors for Mycobacterium ulcerans infection, southeastern Australia. Emerg Infect Dis. 2007;13:1661-6. doi:10.3201/ eid1311.061206.

67. Johnson PDR, Azuolas J, Lavender CJ, Wishart E, Stinear TP, Hayman JA, et al. Mycobacterium ulcerans in mosquitoes captured during outbreak of buruli ulcer, Southeastern Australia. Emerg Infect Dis. 2007;13:1653-60. doi:10.3201/eid1311.061369.

68. Lavender CJ, Fyfe JAM, Azuolas J, Brown K, Evans RN, Ray LR, et al. risk of buruli ulcer and detection of Mycobacterium ulcerans in mosquitoes in Southeastern Australia. PLoS Negl Trop Dis. 2011;5:e1305. doi:10.1371/journal.pntd.0001305.

69. Wallace JR, Gordon MC, Hartsell L, Mosi L, Benbow ME, Merritt $\mathrm{RW}$, et al. Interaction of Mycobacterium ulcerans with mosquito species: implications for transmission and trophic relationships. Appl Environ Microbiol. 2010;76:6215-22. doi:10.1128/AEM. 00340-10.

70. Vogler AJ, Chan F, Wagner DM, Roumagnac P, Lee J, Nera R, et al. Phylogeography and molecular epidemiology of yersinia pestis in Madagascar. PLoS Negl Trop Dis. 2011;5:e1319. doi:10.1371/ journal.pntd.0001319.

71. Portaels F, Elsen P, Guimaraes-Peres A, Fonteyne PA, Meyers WM. Insects in the transmission of Mycobacterium ulcerans infection. Lancet. 1999;353:986. doi:10.1016/S0140-6736(98)05177-0.

72. Jacobsen KH, Padgett JJ. Risk factors for Mycobacterium ulcerans infection. Int J Infect Dis. 2010;14:e677-81. doi:10.1016/j.ijid. 2009.11.013.

73. Einarsdottir T, Huygen K. Buruli ulcer. Hum Vaccines. 2011;7: 1198-203. doi:10.4161/hv.7.11.17751.

74. Silva MT, Portaels F, Pedrosa J. Pathogenetic mechanisms of the intracellular parasite Mycobacterium ulcerans leading to Buruli ulcer. Lancet Infect Dis. 2009;9:699-710. doi:10.1016/S14733099(09)70234-8.

75. Coutanceau E, Marsollier L, Brosch R, Perret E, Goossens P, Tanguy M, et al. Modulation of the host immune response by a transient intracellular stage of Mycobacterium ulcerans: the contribution of endogenous mycolactone toxin. Cell Microbiol. 2005;7: 1187-96. doi:10.1111/j.1462-5822.2005.00546.x.

76. Torrado E, Fraga AG, Castro AG, Stragier P, Meyers WM, Portaels $F$, et al. Evidence for an intramacrophage growth phase of Mycobacterium ulcerans. Infect Immun. 2007;75:977-87. doi:10. 1128/IAI. 00889-06.

77. Schütte D, Pluschke G. Immunosuppression and treatmentassociated inflammatory response in patients with Mycobacterium ulcerans infection (Buruli ulcer). Expert Opin Biol Ther. 2009;9: 187-200. doi:10.1517/14712590802631854.

78. Hamer SA, Lehrer E, Magle SB. Wild birds as sentinels for multiple zoonotic pathogens along an urban to rural gradient in Greater Chicago, Illinois. Zoonoses Pub Health. 2012;59:355-64. doi:10. 1111/j.1863-2378.2012.01462.x. 